\title{
A new closed form for Libby-Novick beta class of generalized distributions with applications to Weibull model
}

\author{
Mohammed Ali Ahmed ${ }^{1 *}$ \\ 1Department of Statistics, Mathematics and Insurance, Al Madina Higher Institute of Management and Technology, Giza, Egypt, \\ mrmohamedali2005@yahoo.com \\ Orcid: 0000-0002-8320-6631 \\ *Corresponding Author, e-mail: mrmohamedali2005@yahoo.com
}

\begin{abstract}
It is useful to derive new classes of distributions having a simple closed form instead of classes having no closed form to get better flexibility in deriving mathematical properties, generating random numbers and applying real data sets. In this paper, a new closed form class of generalized distributions, so-called the modified Libby-Novick (MLN) class, is derived from the implicit form of Libby-Novick beta class. Two important classes of distributions are nested by the MLN class. Some generalized mathematical properties are derived and the MLN class parameters estimation using maximum likelihood estimation (MLE) method is obtained. A simulation study using bootstrapping approach is applied to investigate the estimators behavior of the MLN-Weibull (MLN-W) distribution. A real data set is used to illustrate the potentiality of the MLN-W
\end{abstract}

Keywords: the new form Libby-Novick distribution, moments, order statistics, maximum likelihood estimation.

\section{Introduction}

Eugene et al. [1] presented for the first time the beta class which has the following CDF and PDF, respectively,

$$
P(y ; \alpha, \beta, W)=\frac{1}{B(\alpha, \beta)} \int_{0}^{G(y ; W)} t^{\alpha-1}(1-t)^{\beta-1} d t ; 0<t<1 ; \alpha, \beta>0 ;-\infty<y<\infty,
$$

and

$$
p(y ; \alpha, \beta, W)=\frac{1}{B(\alpha, \beta)} g(y ; W) G(y ; W)^{\alpha-1}\{1-G(y ; W)\}^{\beta-1},
$$

where $G(y ; W)$ is the $C D F$ of the baseline distribution, $W$ is the parameter vector of the baseline distribution. Wahed [2] presented a general method for constructing an extended class of generalized distributions by using the following $C D F$ 


$$
F(\mathrm{y} ; T, W)=\int_{0}^{G(\mathrm{y} ; W)} g(\mathrm{t} ; \mathrm{T}) d t ; 0<t<1,
$$

where $G(\mathrm{y} ; W)$ is the $C D F$ of the baseline distribution and $g(t ; T)$ is the PDF of the generator distribution, $T$ is the parameter vector of the generator distribution and $W$ is the parameter vector of the baseline distribution. Based on [2] many classes of generalized distributions are derived as the Kumaraswamy $(K W)$ class, [3] and [4], the Kummer beta class [5], the McDonald class, [6] and [7], the Kumaraswamy- Kumaraswamy $(K W-K W)$ class, [8] and [9], the Libby-Novick beta class, [10] and [11], which have the following $C D F$ and $P D F$

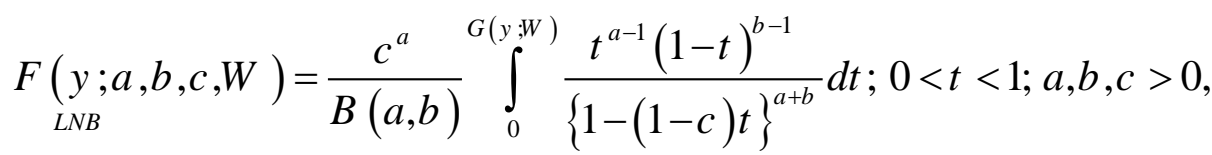

and

$$
f \underset{L N B}{(y ; a, b, c, W)}=\frac{c^{a} g(x ; W) G(x ; W)^{a-1}\{1-G(x ; W)\}^{b-1}}{B(a, b)\{1-(1-c) G(x ; W)\}^{a+b}} .
$$

The main goal of this study is to derive a new explicit form class of distributions depending on the new form Libby-Novick distribution, Ali Ahmed [12], as a simple generator.

The rest of this paper is organized as follows: In section 2, the proposed class is derived. In section 3 , some mathematical properties are given. In section 4, the Hazard function is obtained. In section 5 , the Rényi entropy is given. In section 6, order statistics are obtained. In Section 7, the MLE method is performed. In Section 8, a simulation study using bootstrapping approach is used. Finally, in Section 9, an application is used practically.

\section{The New MLN Class of Generalized Distributions}

Ali Ahmed [12] presented, for the first time, the new form Libby-Novick (NLN) distribution where he derived the NLN distribution using a transformation into the Libby- Novick beta distribution, the NLN distribution is much simpler to use than Libby-Novick beta distribution, in simulation studies or in mathematical properties, because of his simple closed form of both its quantile function and cumulative distribution function.

The NLN distribution has the following CDF and PDF, respectively,

$$
G(t ; \alpha, \beta, c)=1-\frac{\left(1-t^{\alpha}\right)^{\beta}}{\left[1-(1-c) t^{\alpha}\right]^{\beta}} ; o<t<1 ; \alpha, \beta, c>0,
$$

and

$$
g(t ; \alpha, \beta, c)=\frac{\alpha \beta c t^{\alpha-1}\left(1-t^{\alpha}\right)^{\beta-1}}{\left[1-(1-c) t^{\alpha}\right]^{\beta+1}},
$$

substituting (3) into (1) gives

$$
F(y ; \alpha, \beta, c, W)=\alpha \beta c \int_{0}^{G(y ; W)} \frac{t^{\alpha-1}\left(1-t^{\alpha}\right)^{\beta-1}}{\left[1-(1-c) t^{\alpha}\right]^{\beta+1}} d t,
$$

setting $x=t^{\alpha}$ gives 


$$
F(y ; \alpha, \beta, c, W)=\beta c \int_{0}^{G^{\alpha}(y ; W)} \frac{(1-x)^{\beta-1}}{[1-(1-c) x]^{\beta+1}} d x,
$$

using the following method of integration, Prudnikov et al. [13],

$\int_{r}^{s} \frac{(1+\delta z)^{\beta-1}}{(1+\zeta z)^{\beta+1}} d z=\frac{(\delta s+1)^{\beta}}{\delta \beta(\zeta s+1)^{\beta}-\beta \zeta(\zeta s+1)^{\beta}}-\frac{(\delta r+1)^{\beta}}{\delta \beta(\zeta r+1)^{\beta}-\beta \zeta(\zeta r+1)^{\beta}} ; \delta, \zeta \in R ; \beta, z>0$,

then,

$$
F(y ; \alpha, \beta, c, W)=\beta c\left\{\frac{1}{\beta c}-\frac{(1-x)^{\beta}}{\beta c(c x-x+1)^{\beta}}\right\}_{0}^{G^{\alpha}(y ; W)},
$$

hence,

$$
F(y ; \alpha, \beta, c, W)=1-\frac{\left[1-G^{\alpha}(y ; W)\right]^{\beta}}{\left[1-(1-c) G^{\alpha}(y ; W)\right]^{\beta}} ;-\infty<y<\infty ; \alpha, \beta, c>0,
$$

differentiating (4) gives, directly, the PDF of the $M L N$ class, as follows,

$$
f(y ; \alpha, \beta, c, W)=\frac{\alpha \beta c g(y ; W) G^{\alpha-1}(y ; W)\left(1-G^{\alpha}(y ; W)\right)^{\beta-1}}{\left[1-(1-c) G^{\alpha}(y ; W)\right]^{\beta+1}},
$$

setting $c=1$ gives the $K W$ class and setting $c=1, \beta=1$ gives the exponentiated $(E X)$ class. Many special distributions can be derived by the MLN class as the MLN-Weibull $(M L N-W)$ distribution, some shapes of the density function for the $M L N-W$ distribution are illustrated in figure 1.

\subsection{An Expansion for the $C D F$ and $P D F$}

Using the binomial expansion for (4) gives

$$
F(y ; \alpha, \beta, c, W)=\alpha \beta c \sum_{i=0}^{\infty}(-1)^{i}\left(\begin{array}{c}
\beta-1 \\
i
\end{array}\right) t^{\alpha i} \int_{0}^{G(y ; W)} \frac{t^{\alpha-1}}{\left[1-(1-c) t^{\alpha}\right]^{\beta+1}} d t,
$$

then,

$$
F(y ; \alpha, \beta, c, W)=\alpha \beta c \sum_{i=0}^{\infty}(-1)^{i}\left(\begin{array}{c}
\beta-1 \\
i
\end{array}\right)^{G(y ; W)} \frac{t^{\alpha i+\alpha-1}}{\left[1-(1-c) t^{\alpha}\right]^{\beta+1}} d t,
$$

substituting $x=t^{\alpha}$ into last equation yields

$$
F(y ; \alpha, \beta, c, W)=\beta c \sum_{i=0}^{\infty}(-1)^{i}\left(\begin{array}{c}
\beta-1 \\
i
\end{array}\right)^{G^{\alpha}(y ; W)} \frac{x^{i+1-1}}{[1-(1-c) x]^{\beta+1}} d x,
$$

using the following method of integration, Gradshteyn and Ryzhik [14],

$$
\int_{0}^{u} \frac{z^{\mu-1}}{[1+\lambda z]^{v}} d z=\frac{u^{\mu}}{\mu}{ }_{2} F_{1}(v, \mu, 1+\mu,-\lambda u),
$$

where $u=G^{\alpha}(y ; W), \mu=i+1, v=\beta+1, \lambda=c-1$, gives 
$F(y ; \alpha, \beta, c, W)=\beta c \sum_{i=0}^{\infty}(-1)^{i}\left(\begin{array}{c}\beta-1 \\ i\end{array}\right) \frac{G^{\alpha(i+1)}(y ; \mathrm{W})}{(i+1)}{ }_{2} F_{1}\left[(\beta+1), i+1, i+2,(1-c) G^{\alpha}(y ; \mathrm{W})\right]$, using the hypergeometric expansion for the last equation yields

$F(y ; \alpha, \beta, c, W)=\beta c \sum_{i=0}^{\infty}(-1)^{i}\left(\begin{array}{c}\beta-1 \\ i\end{array}\right) \frac{G^{\alpha(i+1)}(y ; W)}{(i+1)} \sum_{j=0}^{\infty} \frac{(\beta+1)_{j}(i+1)_{j}}{(i+2)_{j}} \frac{\left[(1-c) G^{\alpha}(y ; W)\right]^{j}}{j !}$,

where $(.)_{j}$ is the ascended factorial, then,

$$
F(y ; \alpha, \beta, c, W)=\beta c \sum_{i, j=0}^{\infty}(-1)^{i}\left(\begin{array}{c}
\beta-1 \\
i
\end{array}\right) \frac{(\beta+1)_{j}(1-c)^{j} G^{\alpha(i+1)+\alpha j}(y ; W)}{j !},
$$

hence,

$$
\begin{aligned}
& F(y ; \alpha, \beta, c, W)=\sum_{i, j=0}^{\infty} m_{i, j} G^{\alpha(i+1)+\alpha j}(y ; W) \\
& m_{i, j}=\beta c(-1)^{i}\left(\begin{array}{c}
\beta-1 \\
i
\end{array}\right) \frac{(\beta+1)_{j}(1-c)^{j}}{j !} .
\end{aligned}
$$

It can be seen that $\alpha$, in the last equation, is a positive integer but one can generalizes the last equation after setting $\alpha$ a positive real, as follows, since

$$
F(y ; \alpha, \beta, c, W)=\sum_{i, j=0}^{\infty} m_{i, j}[1-(1-G(y ; W))]^{\alpha(i+1)+\alpha j},
$$

using the binomial expansion twice for the last equation gives

$$
F(y ; \alpha, \beta, c, W)=\sum_{i, j=0}^{\infty} m_{i, j} \sum_{k=0}^{\infty}(1-G(y ; W))^{k}(-1)^{k}\left(\begin{array}{c}
(i+1)+\alpha j \\
k
\end{array}\right),
$$

then,

$$
F(y ; \alpha, \beta, c, W)=\sum_{i, j=0}^{\infty} m_{i, j} \sum_{k=0}^{\infty}(-1)^{k}\left(\begin{array}{c}
(i+1)+\alpha j \\
k
\end{array}\right) \sum_{p=0}^{k} G^{p}(y ; W)(-1)^{p}\left(\begin{array}{c}
k \\
p
\end{array}\right),
$$

since,

$$
\sum_{k=0}^{\infty} \sum_{p=0}^{k}=\sum_{p=0}^{\infty} \sum_{k=p}^{\infty}
$$

hence,

$$
\begin{aligned}
& F(y ; \alpha, \beta, c, W)=\sum_{p=0}^{\infty} Q_{p} G^{p}(y ; W) \\
& Q_{p}=\sum_{i, j=0}^{\infty} \sum_{k=p}^{\infty} m_{i, j}(-1)^{k}\left(\begin{array}{c}
(i+1)+\alpha j \\
k
\end{array}\right)(-1)^{p}\left(\begin{array}{l}
k \\
p
\end{array}\right),
\end{aligned}
$$

differentiating (8) yields 


$$
f(y ; \alpha, \beta, c, W)=\sum_{p=1}^{\infty} p Q_{p} g(y ; W) G^{p-1}(y ; W)
$$

shifting the index p backward yields

$$
f(y ; \alpha, \beta, c, W)=\sum_{p=0}^{\infty}(p+1) Q_{p+1} g(y ; W) G^{p}(y ; W),
$$

hence,

$$
f(y ; \alpha, \beta, c, W)=\sum_{p=0}^{\infty} Q_{p}^{*} g(y ; W) G^{p}(y ; W),
$$

where,

$$
Q_{p}^{*}=(p+1) Q_{p+1}
$$

\subsection{The Condition for the PDF Expansion}

since,

$$
\sum_{p=0}^{\infty} Q_{p}^{*} \int_{-\infty}^{\infty} \mathrm{g}(y ; W) G^{p}(y ; W) d y=1,
$$

then,

$$
\sum_{p=0}^{\infty} Q_{p}^{*}\left[\frac{G^{p+1}(y ; W)}{(p+1)}\right]_{-\infty}^{\infty}=1
$$

hence,

$$
\sum_{p=0}^{\infty} \frac{Q_{p}^{*}}{p+1}=1
$$

\section{Some Properties of the MLN Class of Distributions}

In this section some properties of the MLN class of distributions will be obtained as follows:

\subsection{The $r$-th Moment}

The random variable $\mathrm{Y}$ having the $P D F$ of the $M L N$ class of distributions has the following $\mathrm{r}$-th moment, Johnson et al.[15], 


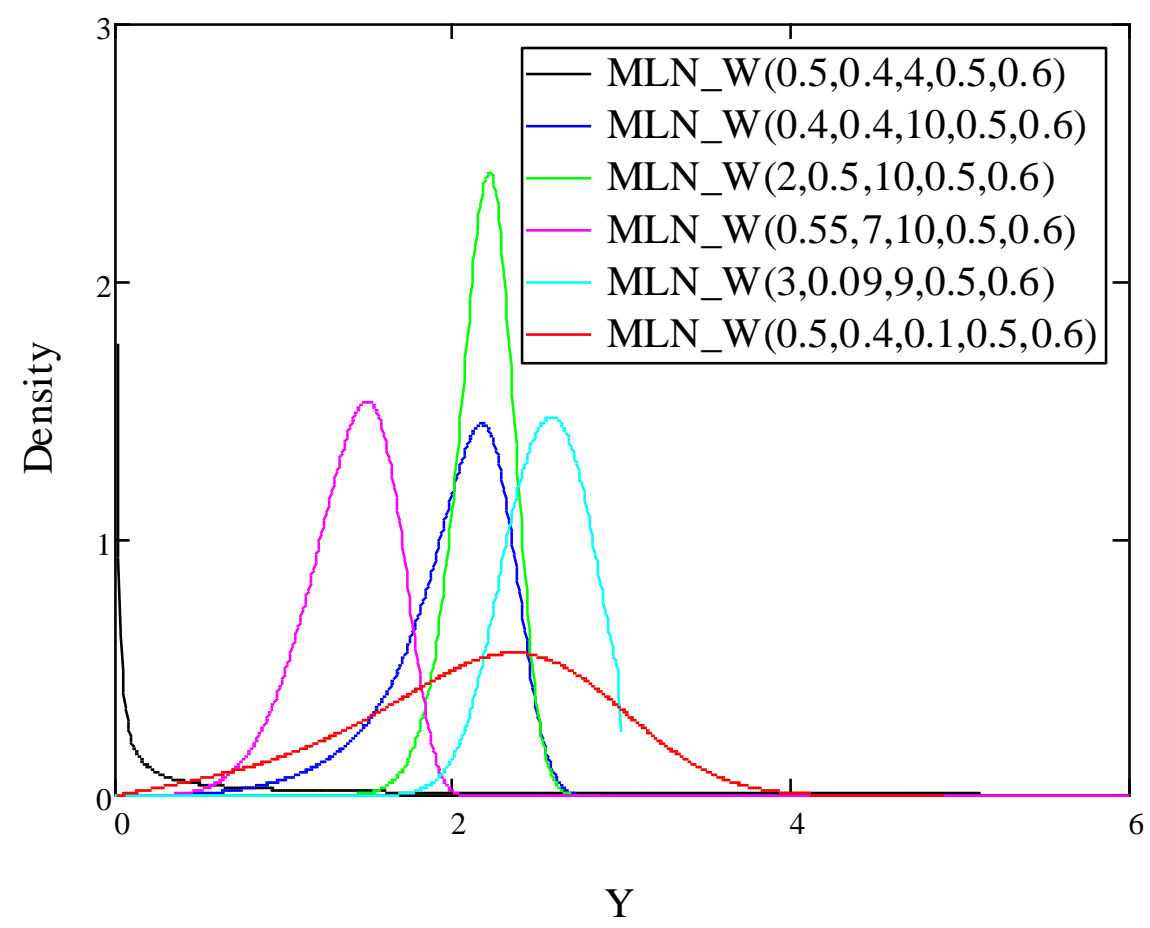

Figure 1: The $M L N-W$ density functions

$$
E\left(y^{r}\right)=\int_{y} y^{r} f(y) d y,
$$

substituting (9) into last equation yields

$$
E\left(y^{r} ; \alpha, \beta, c, W\right)=\sum_{p=0}^{\infty} Q_{p}^{*} \int_{-\infty}^{\infty} y^{r} g(y ; W) G^{p}(y ; W) d y,
$$

then,

$$
E\left(y^{r} ; \alpha, \beta, c, W\right)=\sum_{p=0}^{\infty} Q_{p}^{*} \tau_{r, p, 0} .
$$

where $\tau$ is the probability weighted moment (PWM), Greenwood et al.[16].

One easily can find that, setting $r=0$ and using (11) gives

$$
E\left(y^{0} ; \alpha, \beta, c, W\right)=\sum_{p=o}^{\infty} Q_{p}^{*} \int_{-\infty}^{\infty} g(y ; W) G^{p}(y ; W) d y,
$$

then,

$$
E\left(y^{0} ; \alpha, \beta, c, W\right)=\sum_{p=o}^{\infty} Q_{p}^{*}\left[\frac{G^{p+1}(y ; W)}{p+1}\right]_{-\infty}^{\infty},
$$

substituting (10) into last equation yields

$$
E\left(y^{0} ; \alpha, \beta, c, W\right)=1 \text {. }
$$




\section{Using the Parent Quantile Function}

Setting $G(y ; W)=u, y=q(u)$ and substituting into (11) gives

$$
E\left(y^{r} ; \alpha, \beta, c, W\right)=\sum_{p=o}^{\infty} Q_{p}^{*} \int_{0}^{1} q^{r}(u) u^{p} d u
$$

then,

$$
E\left(y^{r} ; \alpha, \beta, c, W\right)=\sum_{p=o}^{\infty} Q_{p}^{*} \tau_{r, p, o} .
$$

\subsection{The PWM}

The random variable $Y$ having the $C D F$ and $P D F$ of the $M L N$ class of distributions has the following PWM, Greenwood et al. [16],

$$
\tau_{r, \mathrm{~s}, 0}=\int_{y} y^{r} f(y) F^{s}(y) d y,
$$

substituting (8) and (9) into last equation gives

$$
\tau_{r, s, o}=\int_{-\infty}^{\infty}\left[\sum_{p=o}^{\infty} Q_{p}^{*} G^{p}(y ; W)\right]\left[\sum_{p=o}^{\infty} Q_{p} G^{p}(y ; W)\right]^{s} y^{r} g(y ; W) d y,
$$

since,

$$
\tau_{r, s, o}=\int_{-\infty}^{\infty}\left[\sum_{p=o}^{\infty} Q_{p}^{*} G^{p}(y ; W)\right]\left[\sum_{p=o}^{\infty} c_{p} G^{p}(y ; W)\right] y^{r} g(y ; W) d y,
$$

where

$$
c_{0}=Q_{0}^{s}, c_{m}=\frac{1}{m Q_{0}} \sum_{p=1}^{m}(p s-m+p) Q_{p} c_{m-p} ; m \geq 1,
$$

hence,

$$
\tau_{r, s, o}=\sum_{p=o}^{\infty} d_{p} \int_{-\infty}^{\infty} y^{r} g(y ; W) G^{p}(y ; W) d y
$$

where

$$
d_{p}=\sum_{p=o}^{n} Q_{p}^{*} c_{n-p}
$$

then,

$$
\tau_{r, s, o}=\sum_{p=o}^{\infty} d_{p} \tau_{r, p, o}
$$




\section{Using the Parent Quantile Function}

Setting $G(y ; W)=u, y=q(u)$ and substituting into (12) yields

$$
\tau_{r, s, o}=\sum_{p=o}^{\infty} d_{p} \int_{0}^{1} q^{r}(u) u^{p} d u
$$

then,

$$
\tau_{r, s, o}=\sum_{p=o}^{\infty} d_{p} \tau_{r, p, o}
$$

\subsection{The Moment Generating Function}

The random variable $Y$ having the $P D F$ of the MLN class of distributions has the following moment generating function $(M G F)$

$$
M_{y}(t)=E\left(e^{t y}\right)=\int_{y} e^{t y} f(y) d y,
$$

using the exponential expansion for last equation gives

$$
E\left(e^{t y}\right)=E\left(\sum_{r=0}^{\infty} \frac{t^{r} y^{r}}{r !}\right)
$$

then,

$$
E\left(e^{t y}\right)=\sum_{r=0}^{\infty} \frac{t^{r} E\left(y^{r}\right)}{r !}
$$

\section{Using the Parent Quantile Function}

Substituting (9) into (13) gives

$$
E\left(e^{t y} ; \alpha, \beta, c, W\right)=\int_{-\infty}^{\infty} e^{t y} \sum_{p=o}^{\infty} Q_{p}^{*} g(y ; W) G^{p}(y ; W) d y,
$$

then,

$$
E\left(e^{t y} ; \alpha, \beta, c, W\right)=\sum_{p=o}^{\infty} Q_{p}^{*} \int_{-\infty}^{\infty} e^{t y} g(y ; W) G^{p}(y ; W) d y
$$

setting $G(y ; W)=u, y=q(u)$ and substituting into last equation yields

$$
E\left(e^{t y} ; \alpha, \beta, c, W\right)=\sum_{p=o}^{\infty} Q_{p}^{*} \int_{0}^{1} e^{t q(u)} u^{p} d u
$$

\subsection{The Mean Deviation}

The random variable $Y$ having the PDF of the $M L N$ class of distributions has the following mean deviation about the mean and about the median, respectively, 


$$
S_{1}(y)=\int_{y}|y-\mu| f(y) d y \quad \text { and } \quad S_{2}(y)=\int_{y}|y-M| f(y) d y,
$$

easily, it can be given by, Ahmed [17], Ali Ahmed [12]

$$
S_{1}(y)=2 \mu F(\mu)-2 t(\mu) \text { and } S_{2}(y)=\mu-2 t(M),
$$

where $T(z)=\int_{-\infty}^{z} y f(y) d y$ is the linear incomplete moment.

Substituting (9) into $T($.) gives

$$
T(z ; \alpha, \beta, c, W)=\int_{-\infty}^{z} y \sum_{p=o}^{n} Q_{p}^{*} g(y ; W) G^{p}(y ; W) d y,
$$

then,

$$
T(z ; \alpha, \beta, c, W)=\sum_{p=o}^{\infty} Q_{p}^{*} \int_{-\infty}^{z} y g(y ; W) G^{p}(y, w) d y .
$$

\section{Using the Parent Quantile Function}

Setting $G(y ; W)=u, y=q(u)$ and substituting into last equation yields

$$
T(z ; \alpha, \beta, c, W)=\sum_{p=o}^{\infty} Q_{p}^{*} \int_{0}^{G(z)} q(u) u^{p} d u
$$

\section{The Hazard Function of the MLN Class of Distributions}

The random variable $Y$ having the $C D F$ of the MLN class of distributions has the following survival function, Meeker and Escobar [18],

$$
S(y)=1-F(y)
$$

substituting (5) into last equation gives

$$
S(y ; \alpha, \beta, c, W)=\frac{\left[1-G^{\alpha}(y ; W)\right]^{\beta}}{\left[1-(1-c) G^{\alpha}(y ; W)\right]^{\beta}} ;-\infty<y<\infty ; \alpha, \beta, c>0 .
$$

Simply, the Hazard function, Meeker and Escobar [18], can be given by

$$
H(y)=\frac{f(y)}{S(y)},
$$

substituting (6) and (14) into last equation yields

$$
H(y ; \alpha, \beta, c, W)=\frac{\alpha \beta c g(y ; W) G(y ; W)^{\alpha-1}}{\left[1-G^{\alpha}(y ; W)\right]\left[1-(1-c) G(y ; W)^{\alpha}\right]},
$$

some shapes of the Hazard function, for example, for the $M L N-W$ distribution are illustrated in figure 2 . 


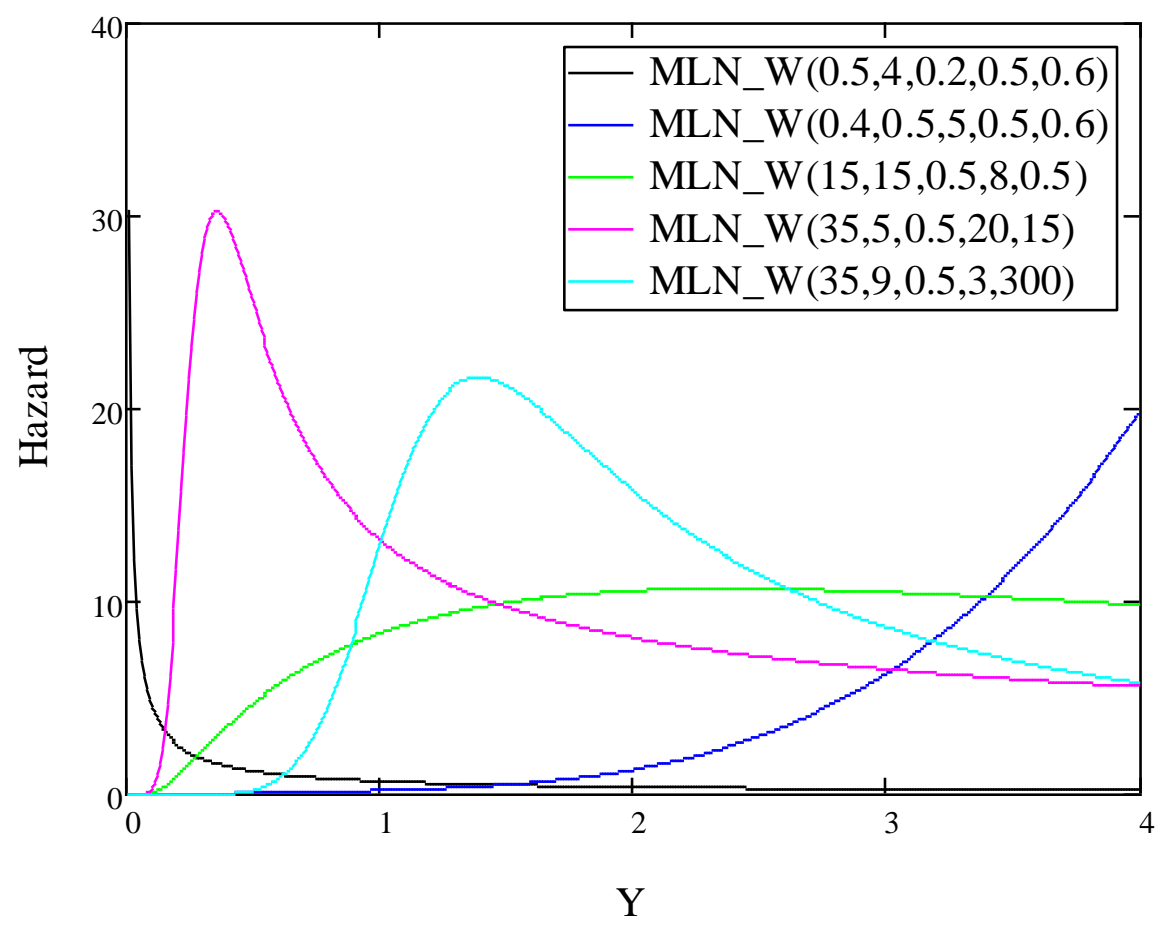

Figure 2: The $M L N-W$ Hazard functions

One can see, in figure 2, three types of Hazard functions curves of the $M L N-W$ distribution are described as follows: A decreasing then constant Hazard curve, an increasing then constant Hazard curve and an increasing then decreasing then constant Hazard curve.

\section{The Rényi Entropy of the MLN Class of Distributions}

The random variable $Y$ having the PDF of the MLN class of distributions has the following Rényi entropy, Meeker and Escobar [18],

$$
e_{R}(\rho)=\frac{1}{1-\rho} \log \left[\int_{-\infty}^{\infty}[f(y)]^{\rho} d y\right],
$$

substituting (9) into last equation gives

$$
e_{R}(\rho)=\frac{1}{1-\rho} \log \left[\int_{-\infty}^{\infty}\left(\sum_{p=0}^{\infty} Q_{p}^{*} g(y ; W) G^{p}(y ; W)\right)^{\rho} d y\right],
$$

then,

$$
e_{R}(\rho)=\frac{1}{1-\rho} \log \left[\int_{-\infty}^{\infty} g^{\rho}(y ; W)\left(\sum_{p=0}^{\infty} Q_{p}^{*} G^{p}(y ; W)\right)^{\rho} d y\right]
$$

hence,

$$
e_{R}(\rho)=\frac{1}{1-\rho} \log \left[\sum_{p=0}^{\infty} a_{p} \int_{-\infty}^{\infty} g^{\rho}(y ; W) G^{p}(y ; W) d y\right],
$$

where, 


$$
a_{0}=\left(Q_{0}^{*}\right)^{\rho}, a_{m}=\frac{1}{m Q_{0}^{*}} \sum_{p=1}^{m}(p \rho-m+p) Q_{p}^{*} a_{m-p} ; m \geq 1 \text {. }
$$

\section{Order Statistics of the MLN Class of Distributions}

A density function $f\left(y_{u: v}\right)$ of the $u$-th order statistics for $u=1,2, \ldots, v$ from iid random variables $Y_{1}, Y_{2}, \ldots, Y_{v}$ following any MLN generalized distribution, Arnold et al.[19], is given by

$$
f\left(y_{u: v}\right)=\frac{f\left(y_{u}\right)}{B(u, v-u+1)} F^{u-1}\left(y_{u}\right)\left\{1-F\left(y_{u}\right)\right\}^{v-u}
$$

using binomial expansion for last equation gives

$$
f\left(y_{u, v}\right)=\sum_{w=0}^{v-u} \frac{(-1)^{w}\left(\begin{array}{c}
v-u \\
w
\end{array}\right)}{\beta(u, v-u+1)} f\left(y_{u}\right) F^{u+w-1}\left(y_{u}\right)
$$

substituting (8) and (9) into last equation gives

$$
\begin{aligned}
f\left(y_{u v} ; \alpha, \beta, c, W\right) & =\sum_{w=0}^{v-u} \frac{(-1)^{w}\left(\begin{array}{c}
v-u \\
w
\end{array}\right)}{\beta(u, v-u+1)}\left[\sum_{p=0}^{\infty} Q_{p}^{*} g\left(y_{u} ; W\right) G^{P}\left(y_{u} ; W\right)\right] \\
& \times\left[\sum_{p=0}^{\infty} Q_{p} G^{P}\left(y_{u} ; W\right)\right]^{u+w-1},
\end{aligned}
$$

then,

$$
f\left(y_{u v} ; \alpha, \beta, c, W\right)=\sum_{w=0}^{v-u} \frac{(-1)^{w}\left(\begin{array}{c}
v-u \\
w
\end{array}\right)}{\beta(u, v-u+1)}\left[\sum_{p=0}^{\infty} Q_{p}^{*} g\left(y_{u} ; W\right) G^{P}\left(y_{u} ; W\right)\right]\left[\sum_{p=0}^{\infty} c_{p} G^{P}\left(y_{u} ; W\right)\right] .
$$

where,

$$
c_{0}=Q_{0}^{u+w-1}, c_{m}=\frac{1}{m Q_{0}} \sum_{p=1}^{m}[p(u+w-1)-m+p] Q_{p} c_{m-p} ; m \geq 1,
$$

hence,

$$
f\left(y_{u v} ; \alpha, \beta, c, W\right)=\sum_{w=0}^{v-u} \frac{(-1)^{w}\left(\begin{array}{c}
v-u \\
w
\end{array}\right)}{\beta(u, v-u+1)} \sum_{p=0}^{\infty} d_{p} g\left(y_{u} ; W\right) G^{P}\left(y_{u} ; W\right)
$$

where,

$$
d_{p}=\sum_{p=o}^{n} Q_{p}^{*} c_{n-p}
$$

moreover, 


$$
f\left(y_{u v} ; \alpha, \beta, c, W\right)=\sum_{p=0}^{\infty} b_{p} g\left(y_{u} ; W\right) G^{P}\left(y_{u} ; W\right)
$$

where,

$$
b_{p}=\sum_{w=0}^{v-u} \frac{(-1)^{w}\left(\begin{array}{c}
v-u \\
w
\end{array}\right)}{\beta(u, v-u+1)} \sum_{p=o}^{n} Q_{p}^{*} c_{n-p} .
$$

The $r$-th moment of order statistics of the MLN class of distributions is given by

$$
E\left(y_{u: v}^{r}\right)=\int_{y_{u}} y_{u}^{r} f\left(y_{u: v}\right) d y_{u},
$$

substituting (15) into last equation yields

$$
E\left(y_{u v}^{r} ; \alpha, \beta, c, W\right)=\sum_{p=o}^{\infty} b_{p} \int_{-\infty}^{\infty} y_{u}^{r} g\left(y_{u} ; W\right) G^{p}\left(y_{u} ; W\right) d y_{u},
$$

so that,

$$
E\left(y_{u v}^{r} ; \alpha, \beta, c, W\right)=\sum_{p=o}^{\infty} b_{p} \tau_{r, p, o}
$$

\section{Estimation for the MLN Class Parameters Using MLE Method}

Let $Y_{1}, Y_{2}, \ldots, Y_{\mathrm{n}}$ be iid random variables following any $M L N$ generalized distribution $(y ; \Lambda)$ then the likelihood function for the vector of parameter $\Lambda=(\alpha, \beta, c, W)$, Garthwait et al.[20], is obtained by

$$
\begin{aligned}
L(y ; \alpha, \beta, c, W) & =(\alpha \beta c)^{n} \prod_{i=1}^{n} g\left(y_{i} ; W\right) \prod_{i=1}^{n} G^{\alpha-1}\left(y_{i} ; W\right) \prod_{i=1}^{n}\left(1-G^{\alpha}\left(y_{i} ; W\right)\right)^{\beta-1} \\
& \times \prod_{i=1}^{n}\left[1-(1-c) G^{\alpha}\left(y_{i} ; W\right)\right]^{-\beta-1},
\end{aligned}
$$

the log likelihood function is given by

$$
\begin{aligned}
\ell(y ; \alpha, \beta, c, W) & =n[\log \alpha+\log \beta+\log c]+\sum_{i=1}^{n} \log g\left(y_{i} ; W\right)+(\alpha-1) \sum_{i=1}^{n} \log G\left(y_{i} ; W\right) \\
& +(\beta-1) \sum_{i=1}^{n} \log \left(1-G^{\alpha}\left(y_{i} ; W\right)\right)+(-\beta-1) \sum_{i=1}^{n} \log \left[1-(1-c) G^{\alpha}\left(y_{i} ; W\right)\right],
\end{aligned}
$$

the score functions for the parameters $\alpha, \beta, \mathrm{c}$ and $W$ are given by

$$
\begin{gathered}
\frac{\partial \ell(y ; \alpha, \beta, c, W)}{\partial \alpha}=\frac{n}{\alpha}+\sum_{i=1}^{n} \log G\left(y_{i} ; W\right)+(1-\beta) \sum_{i=1}^{n} \frac{G^{\alpha}\left(y_{i} ; W\right) \log G\left(y_{i} ; W\right)}{1-G^{\alpha}\left(y_{i} ; W\right)} \\
+(1+\beta) \sum_{i=1}^{n} \frac{(1-c) G^{\alpha}\left(y_{i} ; W\right) \log G\left(y_{i} ; W\right)}{1-(1-c) G^{\alpha}\left(y_{i} ; W\right)}
\end{gathered}
$$




$$
\begin{aligned}
& \frac{\partial \ell(y ; \alpha, \beta, c, W)}{\partial \beta}=\frac{n}{\beta}+\sum_{i=1}^{n} \log \left(1-G^{\alpha}\left(y_{i} ; W\right)\right)-\sum_{i=1}^{n} \log \left[1-(1-c) G^{\alpha}\left(y_{i} ; W\right)\right], \\
& \frac{\partial \ell(y ; \alpha, \beta, c, W)}{\partial c}=\frac{n}{c}-(\beta+1) \sum_{i=1}^{n} \frac{G^{\alpha}\left(y_{i} ; W\right)}{\left[1-(1-c) G^{\alpha}\left(y_{i} ; W\right)\right]},
\end{aligned}
$$

and

$$
\begin{aligned}
\frac{\ell(y ; \alpha, \beta, c, W)}{\partial w_{j}} & =\sum_{i=1}^{n} \frac{1}{g\left(y_{i} ; W\right)} \frac{\partial g\left(y_{i} ; W\right)}{\partial w_{j}}+(\alpha-1) \sum_{i=1}^{n} \frac{1}{G\left(y_{i} ; W\right)} \frac{\partial G\left(y_{i} ; W\right)}{\partial w_{j}} \\
& +(1-\beta) \sum_{i=1}^{n} \frac{\alpha G^{\alpha-1}\left(y_{i} ; W\right)}{1-G^{\alpha}\left(y_{i} ; W\right)} \frac{\partial G\left(y_{i} ; W\right)}{\partial w_{j}} \\
& +(1+\beta) \sum_{i=1}^{n} \frac{(1-c) \alpha G^{\alpha-1}\left(y_{i} ; W\right)}{1-(1-c) G^{\alpha}\left(y_{i} ; W\right)} \frac{\partial G\left(y_{i} ; W\right)}{\partial w_{j}} .
\end{aligned}
$$

\section{A Simulation Study}

This study aims to obtain MLEs of the $M L N-W$ distribution parameters via random numbers to study the sample behavior of MLEs by the bootstrapping resample approach.

Obtaining parameters estimates algorithm is detailed in the following steps:

i. Generating a random sample $Y_{1}, Y_{2}, \ldots, Y_{n}$ having sizes $n=(5,15,30,50,100,300)$ using the $M L N-W$ distribution.

ii. Selecting different parameters set values as: $\operatorname{set}(1)$ : $(\alpha=0.1, \beta=0.2, \theta=0.3, \lambda=4, c=3)$, set (2): $(\alpha=0.1, \beta=0.2$, $\theta=0.3, \lambda=6, c=3)$ and set (3): $(\alpha=0.1, \beta=0.2, \theta=0.5, \lambda=4, c=3)$.

iii. Solving the $M L N-W$ distribution normal equations by iteration to estimate distribution parameters.

iv. Replacing set (1), set (2) and set (3) with its estimators and repeating step (3) to calculate: Biases, MLES, RMSE (the root of mean squared error) and the Pearson type of parameters estimators of the $M L N-W$ distribution, Pearson (1895).

v. Repeating step (1) to step (4), 10000 times.

Random numbers samples are generated via Mathcad package v15 using the conjugate gradient iteration method. All outcomes are indicated in the appendix.

Clearly, from study results included in the appendix, Biases and RMSEs decrease as sample size increases. In all times, $\hat{\beta}$ and $\hat{\lambda}$ sampling distributions follow the Pearson type IV distribution, $\hat{\alpha}$, $c$ and $\hat{\theta}$ sampling distributions differ according to sample size. As $\lambda$ increases, for fixed values of $\alpha, \beta, c$ and $\theta$, the biases and MSEs of $\hat{\beta}$ and $\hat{\theta}$ decrease.. Also, one can see that when sample size increases, the estimators can be consistent.

\section{Application}

A real data set is used, practically, to investigate the flexibility of the new model using $M L E$ method via the Mathematica package version 10, some distributions are used as: the $M L N-W$ distribution, the gamma distribution, 
the Gumbel (Max) distribution, the Singh-Maddala distribution, Kumar [21], the Kumaraswamy-Weibull $(K W-W)$ distribution, Cordeiro et al. [22], the exponentiated-Weibull (EX-W) distribution, Nassar et al. [23], and the Weibull $(W)$ distribution. The lifetime (Hours) of classic lamps for 60 devices as follows, given from the $U K$ National Physical Laboratory at http://www.npl.co.uk/

$3.337,0.988,6.058,0.347,0.924,2.484,3.149,0.478,5.000,5.273,5.600,0.348,7.208,3.087,2.405,2.123$, $1.023,2.154,4.535,1.164,1.330,0.494,0.164,4.766,5.396,3.338,4.663,3.124,7.406,4.494,3.136,8.305$, $0.483,3.623,0.060,1.761,3.862,2.451,2.714,4.937,3.343,2.225,0.608,5.151,1.950,5.504,0.244,3.860$, $4.431,0.434,0.083,2.456,1.886, \quad 1.396,2.823, \quad 0.430, \quad 0.573, \quad 4.499, \quad 0.260, \quad 0.634$.

Some measures for goodness of fit and likelihood ratio tests are obtained and included in table (1) and table (2), respectively, the figure (3) shows PDFs for some distributions having skewness and kurtosis values similar to the $M L N-W$ distribution (the gamma distribution, the Gumbel (Max) distribution, the Singh-Maddala distribution) and the figure (4) shows the empirical CDF compared to CDFs for some distributions (the gamma distribution, the Gumbel (Max) distribution, the Singh-Maddala distribution) and the figure (5) illustrates probability density functions for special cases from the $M L N-W$ distribution (the $K W-W$ distribution, the $E X-W$ distribution and the $W$ distribution).

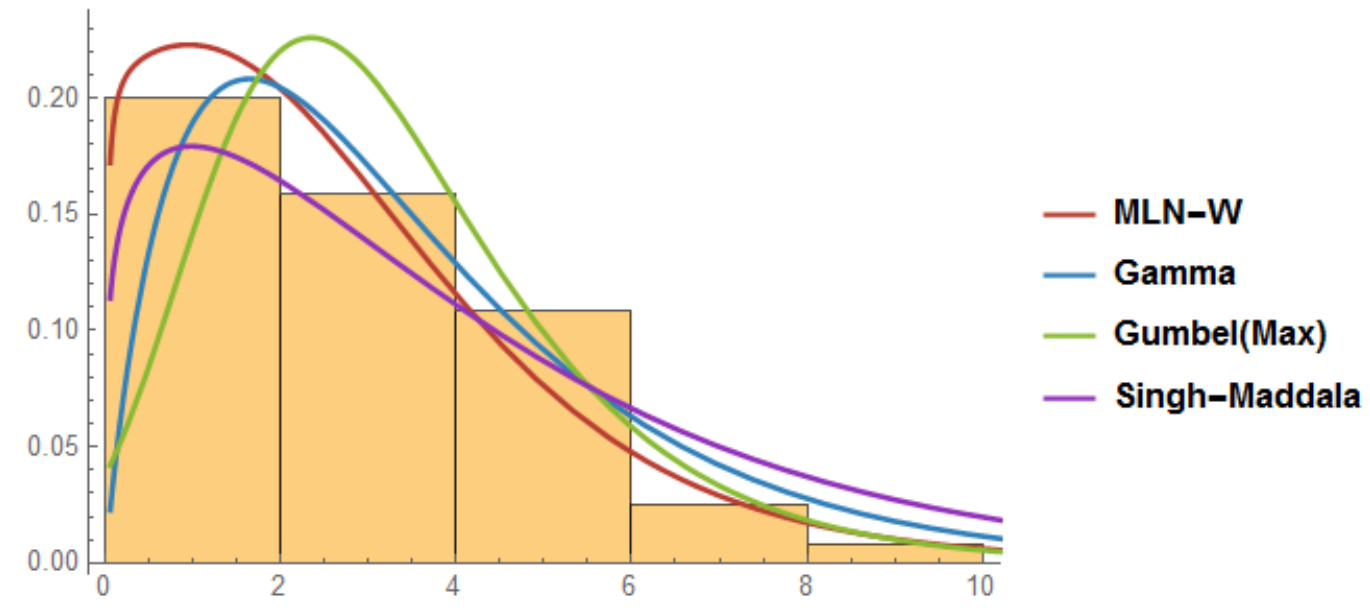

Figure 3: Probability density functions for some distributions having skewness and kurtosis values similar to the $M L N-W$ distribution

Table 1: The MLE of the parameter(s) and the associated AIC and BIC values.

\begin{tabular}{|c|c|c|c|c|c|c|c|c|c|c|c|c|c|}
\hline \multirow{2}{*}{ 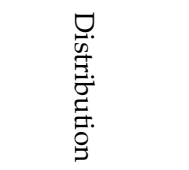 } & \multicolumn{5}{|c|}{ MLE_parameters } & \multirow{2}{*}{ 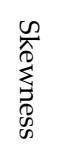 } & \multirow{2}{*}{ 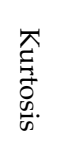 } & \multirow{2}{*}{$K S$} & \multirow{2}{*}{ P-value } & \multirow{2}{*}{ 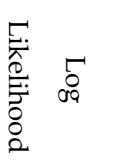 } & \multirow{2}{*}{$A I C$} & \multirow{2}{*}{$B I C$} & \multirow{2}{*}{ CAIC } \\
\hline & $\alpha$ & $\beta$ & c & $\Lambda$ & $\theta$ & & & & & & & & \\
\hline \multirow[t]{2}{*}{$M L N \_W$} & 3.103 & 2.012 & 0.110 & 0.503 & 3.101 & 1.489 & 6.331 & 0.119 & 0.327 & -119.502 & 249.003 & 259.475 & 250.114 \\
\hline & $(0.142)$ & $(0.107)$ & $(0.012)$ & $(0.121)$ & $(0.725)$ & & & & & & & & \\
\hline \multirow[t]{2}{*}{ Singh-Maddla } & 3.3 & 1.2088 & 5.1 & & & 1.505 & 4.159 & 0.174 & 0.044 & -124.692 & 255.383 & 261.666 & 255.812 \\
\hline & $(0.154)$ & $(0.147)$ & $(1.812)$ & & & & & & & & & & \\
\hline Gamma & 1.8772 & 1.8662 & & & & 1.459 & 5.196 & 0.190 & 0.021 & -127.138 & 258.275 & 262.464 & 258.486 \\
\hline
\end{tabular}


$(0.201) \quad(0.153)$

Gumbel

(Max)

In table (1): MLEs of distributions parameters, test statistic of Kolmogorov-Smirnov (KS), the corresponding RMSE (given in parentheses), AIC (Akaike Information Criterion), BIC (Bayesian information criterion) and CAIC (the consistent Akaike Information Criterion), Merovcia and Puka [24], are computed for all distributions having skewness and kurtosis values similar to the $M L N-W$ distribution as the gamma distribution, the Gumbel (Max) distribution and the Singh-Maddala distribution. The null hypothesis is the data follow the $M L N-W$ distribution and it can be accepted at significance level $\alpha=0.05$ where the $M L N-W$ distribution has the smallest $K S, A I C, C A I C, B I C, S E S$ and the largest $\mathrm{p}$-value, so that, the $M L N-\mathrm{W}$ distribution can be the best fitted distribution to the data between other distributions.

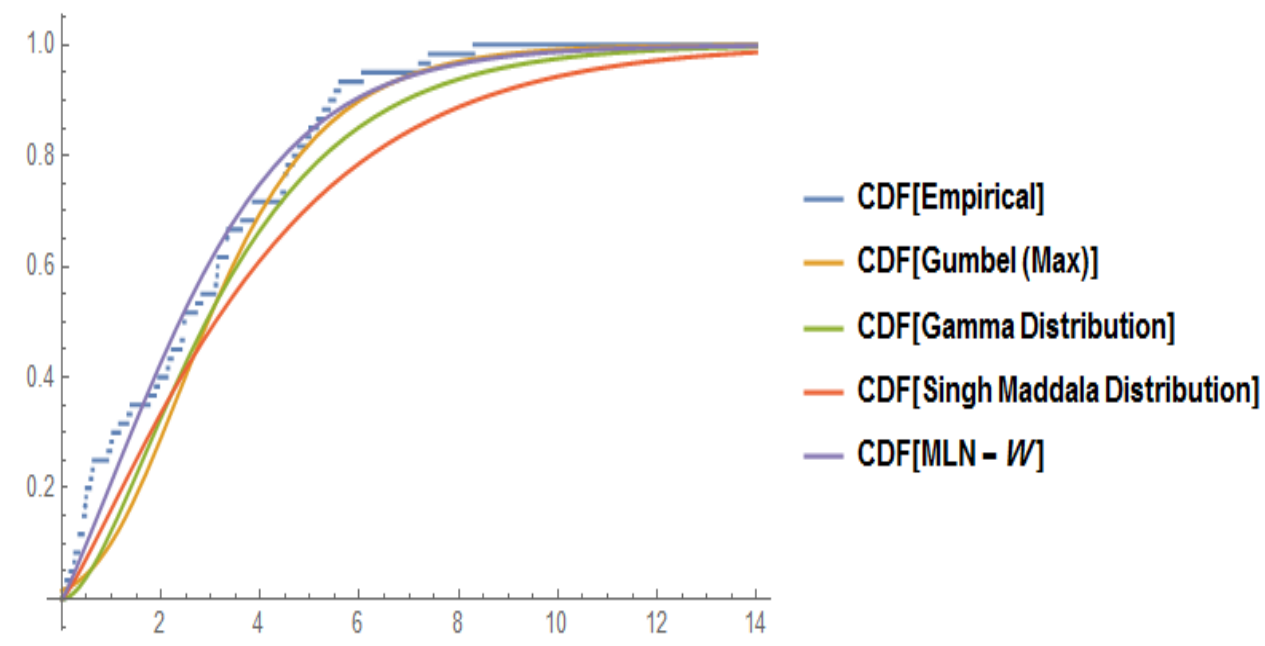

Figure 4: The empirical $C D F$ compared to $C D F$ s for some distributions

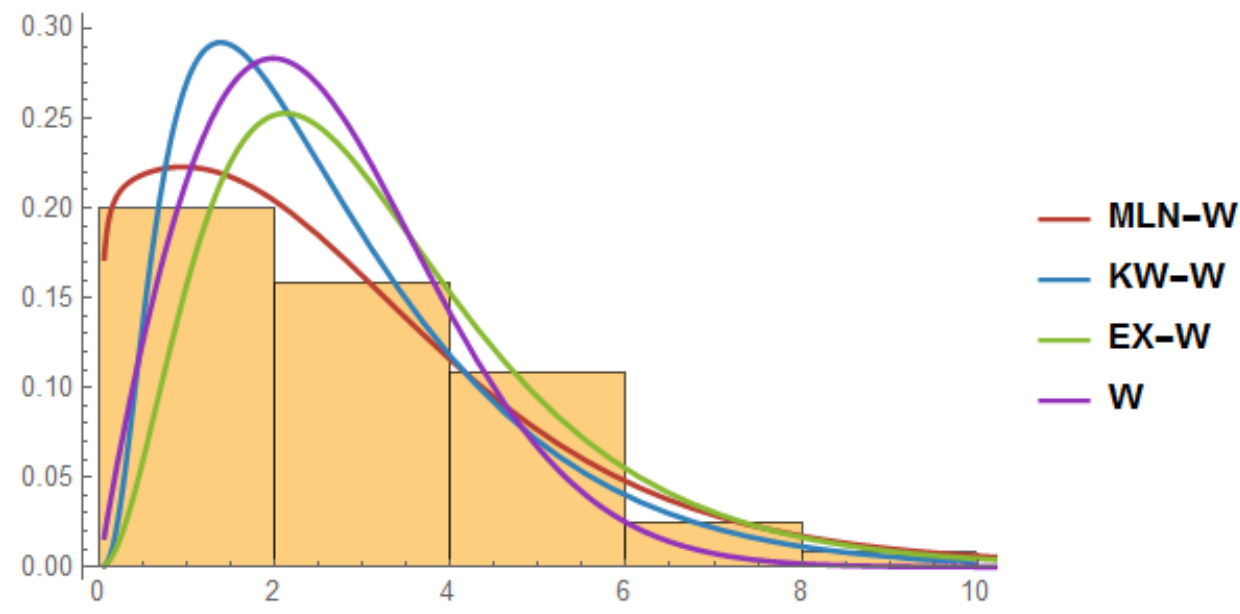

Figure 5: Probability density functions for special cases from the $M L N-W$ distribution 
Table 2: The log-likelihood function, the likelihood ratio tests statistic and p-values.

\begin{tabular}{|c|c|c|c|c|c|c|c|c|c|}
\hline \multirow[b]{2}{*}{ Distribution } & \multicolumn{5}{|c|}{ Parameters } & \multirow[b]{2}{*}{ likelihood) } & \multirow{2}{*}{$\begin{array}{c}\Lambda \\
\text { (The } \\
\text { likelihood } \\
\text { ratio } \\
\text { test statistic) }\end{array}$} & \multirow{2}{*}{$\begin{array}{c}\text { df } \\
\text { degrees( } \\
\text { of } \\
\text { freedom) }\end{array}$} & \multirow[b]{2}{*}{ p-value } \\
\hline & $\alpha$ & $\beta$ & $\mathrm{c}$ & $\Theta$ & $\lambda$ & & & & \\
\hline \multirow[t]{2}{*}{$K W-W$} & 2.985 & 0.151 & - & 1.298 & 1.632 & -137.534 & 36.064 & 1 & $1.909 \times 10^{-9}$ \\
\hline & $(0.149)$ & $(0.118)$ & & $(0.134)$ & $(0.709)$ & & & & \\
\hline \multirow[t]{2}{*}{$E X-W$} & 2.774 & - & - & 1.099 & 0.506 & -143.153 & 47.302 & 2 & $5.352 \times 10^{-11}$ \\
\hline & $(0.152)$ & & & $(0.221)$ & $(0.947)$ & & & & \\
\hline \multirow[t]{2}{*}{$W$} & - & - & - & 1.898 & 0.341 & -133.532 & 28.06 & 3 & $3.528 \times 10^{-6}$ \\
\hline & & & & $(0.129)$ & $(0.737)$ & & & & \\
\hline
\end{tabular}

*Note that the log likelihood of the $M L N-W$ distribution $=-119.502$

In table 2, upon the likelihood ratio test, the null hypothesis is the data follow the nested model and it can be rejected at the level of significance $\alpha=0.05$, so the $M L N-W$ distribution can fit the data better than all nested distributions at significance level $\alpha=0.05$, where the $K W-W$ distribution, the $E X-W$ distribution and the $W$ distribution are nested by $M L N-W$ distribution.

\section{Conclusion}

The modified Libby-Novick class of generalized distributions has several advantages as: it does not have any special function having implicit form, has flexible mathematical properties, simple quantile function and generalizes two important classes of distributions (the $K W$ class and the EX class). The maximum likelihood estimation method is used easily to estimate the MLN class parameters, the $M L N-W$ distribution works practically well when it be compared with other distributions. The author encourages researchers to do more researches and applications on the MLN class of generalized distributions.

Conflicts of Interest: The author has no conflicts of interest.

Funding: No financial resources were provided for this study.

Acknowledgements: The author thanks anyone suggested any important advices or helpful provided comments for this manuscript.

\section{References}

[1] Eugene, N., Lee, C. \& Famoye, F. (2002). Beta-Normal Distribution and Its Applications. Communications in Statistics, Theory and Methods, 31, 497-512. 
[2] Wahed, A.S. (2006). A General Method of Constructing Extended Families of Distribution from an Existing Continuous Class. Journal of Probability and Statistical Science, 4, 165-177.

[3] Kumaraswamy, P. (1980). Generalized Probability Density Function for Double-Bounded Random-Processes. Journal of Hydrology, 46, 79-88.

[4] Cordeiro, GM \& de Castro, M. (2011). A New Family of Generalized Distributions. Journal of Statistical Computation \& Simulation, 81, 883-898.

[5] Pescim, R. R., Cordeiro, G. M., Demétrio, C. G., Ortega, E. M. \& Nadarajah, S. (2012). The new class of Kummer beta generalized distributions. SORT-Statistics and Operations Research Transactions, 153-180.

[6] McDonald, J.B. (1984). Some generalized functions for the size distribution of income. Econometrica, 52, 647-664.

[7] Alexander, C., Cordeiro, G.M., Ortega, E.M.M. \& Sarabia, J.M. (2012). Generalized beta-generated distributions. Comput Stat Data Anal, 56, 1880-1897.

[8] El-Sherpieny, E.A. \& Ahmed, MA. (2014). On The Kumaraswamy Kumaraswamy Distribution. International Journal of Basic and Applied Sciences, 3, 372-381

[9] Mahmoud, M.R., El-Sherpieny, E.A. \& Ahmed, M.A. (2015). The New Kumaraswamy Kumaraswamy Family of Generalized Distributions with Application. Pakistan Journal of Statistics and Operations Research, 11, 159-180.

[10] Libby, D.L. \& Novick, M.R. (1982). Multivariate generalized beta-distributions with applications to utility assessment. Journal of Educational Statistics, 7, 271-294.

[11] Cordeiro, G.M., de Santana, L.H. \& Ortega, E.M., Pescim, R.R.(2014). A new family of distributions: Libby-Novick beta. International Journal of Statistics and Probability, 3, 63-80.

[12] Ali Ahmed, M. (2021). The new form Libby-Novick distribution. Communications in Statistics-Theory and Methods, 50, 540-559.

[13] Prudnikov, A.P., Brychkov, Y.A. \& Marichev, O.I. (1986). Integrals and Series. Gordon and Breach Science Publishers, Amsterdam.

[14] Gradshteyn, I.S. \& Ryzhik, I.M. (2000). Tables of Integrals, Series, and Products. Academic Press, San Diego, CA.

[15] Johnson, N.L., Kotz, S. \& Balakrishnan, N.(1995). Continuous Univariate Distributions. John wiley and Sons, New York.

[16] Greenwood, J.A., Landwehr, J.M., Matalas, N.C. \& Wallis, J.R. (1979). Probability Weighted Moments Definition and Relation to Parameters of Several Distributions. Expressable in Inverse Form. Water Resources Research. 15, 1049-1054.

[17] Ahmed, M.A. (2020). On the alpha power Kumaraswamy distribution: Properties, simulation and application. Revista Colombiana de Estadística. 43, 285-313.

[18] Meeker, W.Q. \& Escobar, L.A. (1998). Statistical Methods for Reliability Data. John Wiley, New York.

[19] Arnold, C.B., Balakrishnan, N. \& Nagaraja, H.N. (1992). A first course in order statistics. John Wiley and Sons, Inc. New York.

[20] Garthwait, P.H., Jolliffe, I.P. \& jones, B. (1995). Statistical Inference. prentice Hall International (UK) Limited, London.

[21] Kumar, D. (2017). The Singh-Maddala distribution: properties and estimation. International journal of system assurance engineering and management, 8, 1297-1311. 
[22] Cordeiro, G.M., Ortega, E.M.M. \& Nadarajah, S. (2010) The Kumaraswamy WeibullDistribution with Application to Failure Data. Journal of The Franklin Institute, 347, 1399-1429.

[23] Nassar, M.M. \& Eissa, F.H. (2003). On the exponentiated Weibull distribution. Communications in Statistics-Theory and Methods, 32, 1317-1336.

[24] Merovcia, F. \& Puka, L. (2014).Transmuted Pareto Distribution. Prob Stat Forum, 7, 1-11.

\section{Appendix}

\begin{tabular}{|c|c|c|c|c|c|c|c|c|}
\hline \multicolumn{9}{|c|}{$\operatorname{Set}(1):(\alpha=0.1, \beta=0.2, \theta=0.3, \lambda=4, c=3)$} \\
\hline $\begin{array}{c}\text { Sample } \\
\text { Size }\end{array}$ & Parameters & $\begin{array}{c}\text { Mean of } \\
\text { Estimators }\end{array}$ & Biases & $\begin{array}{c}\text { Total } \\
\text { Bias }\end{array}$ & RMSE & $\begin{array}{l}\text { Total } \\
\text { RMSE }\end{array}$ & $\begin{array}{l}\text { Pearson } \\
\text { System } \\
\text { Coefficients }\end{array}$ & $\begin{array}{c}\text { Pearson } \\
\text { Type }\end{array}$ \\
\hline \multirow[t]{5}{*}{10} & $\alpha=0.1$ & 0.053 & -0.047 & 2.127 & 0.065 & 5.443 & -0.538 & $\mathrm{I}$ \\
\hline & $\beta=0.2$ & 0.304 & 0.104 & & 0.235 & & 0.501 & IV \\
\hline & $\theta=0.3$ & 0.341 & 0.041 & & 0.091 & & -0.012 & I \\
\hline & $\lambda=4$ & 4.935 & 0.935 & & 4.577 & & 0.81 & IV \\
\hline & $c=3$ & 1.093 & -1.907 & & 2.934 & & 0.346 & IV \\
\hline \multirow[t]{5}{*}{20} & $\alpha=0.1$ & 0.067 & -0.033 & 2.034 & 0.056 & 4.309 & 0.295 & IV \\
\hline & $\beta=0.2$ & 0.282 & 0.082 & & 0.083 & & 0.238 & IV \\
\hline & $\theta=0.3$ & 0.331 & 0.031 & & 0.049 & & 0.134 & IV \\
\hline & $\lambda=4$ & 4.862 & 0.862 & & 3.138 & & 0.302 & IV \\
\hline & $c=3$ & 1.160 & -1.84 & & 2.952 & & 0.391 & IV \\
\hline \multirow[t]{5}{*}{30} & $\alpha=0.1$ & 0.075 & -0.025 & 1.505 & 0.056 & 3.862 & -0.314 & I \\
\hline & $\beta=0.2$ & 0.247 & 0.047 & & 0.037 & & 0.097 & IV \\
\hline & $\theta=0.3$ & 0.33 & 0.03 & & 0.042 & & -0.131 & I \\
\hline & $\lambda=4$ & 4.730 & 0.730 & & 2.482 & & 0.78 & IV \\
\hline & $c=3$ & 1.685 & -1.315 & & 2.957 & & 0.407 & IV \\
\hline \multirow[t]{5}{*}{50} & $\alpha=0.1$ & 0.086 & -0.014 & 1.088 & 0.058 & 3.599 & 0.332 & IV \\
\hline & $\beta=0.2$ & 0.235 & 0.035 & & 0.027 & & 0.085 & IV \\
\hline & $\theta=0.3$ & 0.321 & 0.021 & & 0.035 & & 0.578 & IV \\
\hline & $\lambda=4$ & 4.511 & 0.511 & & 2.038 & & 0.324 & IV \\
\hline & $c=3$ & 2.04 & -0.96 & & 2.965 & & 0.527 & IV \\
\hline \multirow[t]{5}{*}{100} & $\alpha=0.1$ & 0.094 & -0.006 & 0.339 & 0.045 & 2.318 & 0.005 & IV \\
\hline & $\beta=0.2$ & 0.210 & 0.010 & & 0.072 & & 0.004 & IV \\
\hline & $\theta=0.3$ & 0.305 & 0.005 & & 0.035 & & -0.076 & I \\
\hline & $\lambda=4$ & 4.269 & 0.269 & & 1.165 & & 0.155 & IV \\
\hline & $c=3$ & 2.794 & -0.206 & & 2.002 & & -0.0004 & I \\
\hline \multirow[t]{5}{*}{300} & $\alpha=0.1$ & 0.096 & -0.004 & 0.025 & 0.025 & 1.133 & 0.003 & IV \\
\hline & $\beta=0.2$ & 0.202 & 0.002 & & 0.037 & & 0.001 & IV \\
\hline & $\theta=0.3$ & 0.300 & 0.000 & & 0.014 & & -0.047 & I \\
\hline & $\lambda=4$ & 4.013 & 0.013 & & 0.561 & & 0.148 & IV \\
\hline & $c=3$ & 2.979 & -0.021 & & 0.984 & & 0.269 & IV \\
\hline
\end{tabular}




\begin{tabular}{|c|c|c|c|c|c|c|c|c|}
\hline \multicolumn{9}{|c|}{$\operatorname{Set}(2):(\alpha=0.1, \beta=0.2, \theta=0.3, \lambda=6, c=3)$} \\
\hline $\begin{array}{c}\text { Sample } \\
\text { Size }\end{array}$ & Parameters & $\begin{array}{c}\text { Mean of } \\
\text { Estimators }\end{array}$ & Biases & $\begin{array}{l}\text { Total } \\
\text { Bias }\end{array}$ & RMSE & $\begin{array}{l}\text { Total } \\
\text { RMSE }\end{array}$ & $\begin{array}{l}\text { Pearson } \\
\text { System } \\
\text { Coefficients }\end{array}$ & $\begin{array}{c}\text { Pearson } \\
\text { Type }\end{array}$ \\
\hline \multirow[t]{5}{*}{10} & $\alpha=0.1$ & 0.056 & -0.044 & 2.407 & 0.127 & 8.031 & -0.903 & I \\
\hline & $\beta=0.2$ & 0.300 & 0.100 & & 0.195 & & 0.588 & IV \\
\hline & $\theta=0.3$ & 0.331 & 0.031 & & 0.083 & & -0.105 & I \\
\hline & $\lambda=6$ & 7.591 & 1.591 & & 7.122 & & 0.943 & IV \\
\hline & $c=3$ & 1.197 & -1.803 & & 3.704 & & 0.881 & IV \\
\hline \multirow[t]{5}{*}{20} & $\alpha=0.1$ & 0.074 & -0.026 & 1.998 & 0.055 & 6.219 & 0.271 & IV \\
\hline & $\beta=0.2$ & 0.281 & 0.081 & & 0.076 & & 0.62 & IV \\
\hline & $\theta=0.3$ & 0.321 & 0.021 & & 0.041 & & -0.064 & I \\
\hline & $\lambda=6$ & 7.319 & 1.319 & & 4.828 & & 0.56 & IV \\
\hline & $c=3$ & 1.501 & -1.499 & & 3.92 & & 1.035 & VI \\
\hline \multirow[t]{5}{*}{30} & $\alpha=0.1$ & 0.084 & -0.016 & 1.463 & 0.057 & 4.927 & 0.278 & IV \\
\hline & $\beta=0.2$ & 0.236 & 0.036 & & 0.031 & & 0.101 & IV \\
\hline & $\theta=0.3$ & 0.320 & 0.020 & & 0.040 & & -0.345 & I \\
\hline & $\lambda=6$ & 7.002 & 1.002 & & 3.939 & & 0.238 & IV \\
\hline & $c=3$ & 1.934 & -1.066 & & 2.959 & & 0.428 & IV \\
\hline \multirow[t]{5}{*}{50} & $\alpha=0.1$ & 0.093 & -0.007 & 0.794 & 0.057 & 4.421 & 0.311 & IV \\
\hline & $\beta=0.2$ & 0.231 & 0.031 & & 0.026 & & 0.094 & IV \\
\hline & $\theta=0.3$ & 0.314 & 0.014 & & 0.032 & & 0.128 & IV \\
\hline & $\lambda=6$ & 6.467 & 0.467 & & 3.286 & & 0.246 & IV \\
\hline & $c=3$ & 2.358 & -0.642 & & 2.958 & & 0.409 & IV \\
\hline \multirow[t]{5}{*}{100} & $\alpha=0.1$ & 0.097 & -0.003 & 0.474 & 0.057 & 3.745 & -0.909 & I \\
\hline & $\beta=0.2$ & 0.205 & 0.005 & & 0.021 & & 0.408 & IV \\
\hline & $\theta=0.3$ & 0.301 & 0.001 & & 0.028 & & 0.269 & IV \\
\hline & $\lambda=6$ & 6.273 & 0.273 & & 2.598 & & 0.47 & IV \\
\hline & $c=3$ & 2.612 & -0.388 & & 2.697 & & -0.486 & I \\
\hline \multirow[t]{5}{*}{300} & $\alpha=0.1$ & 0.099 & -0.001 & 0.061 & 0.037 & 1.392 & 0.105 & IV \\
\hline & $\beta=0.2$ & 0.201 & 0.001 & & 0.018 & & 0.643 & IV \\
\hline & $\theta=0.3$ & 0.300 & 0.000 & & 0.010 & & -0.57 & I \\
\hline & $\lambda=6$ & 6.053 & 0.053 & & 0.618 & & 0.671 & IV \\
\hline & $c=3$ & 2.968 & -0.032 & & 1.247 & & 0.347 & IV \\
\hline
\end{tabular}

$\operatorname{Set}(3):(\alpha=0.1, \beta=0.2, \theta=0.5, \lambda=4, c=3)$

\begin{tabular}{ccccccccc}
\hline $\begin{array}{c}\text { Sample } \\
\text { Size }\end{array}$ & Parameters & $\begin{array}{c}\text { Mean of } \\
\text { Estimators }\end{array}$ & Biases & $\begin{array}{c}\text { Total } \\
\text { Bias }\end{array}$ & RMSE & $\begin{array}{c}\text { Total } \\
\text { RMSE }\end{array}$ & $\begin{array}{l}\text { Pearson } \\
\text { System } \\
\text { Coefficients }\end{array}$ & $\begin{array}{c}\text { Pearson } \\
\text { Type }\end{array}$ \\
\hline 10 & $\alpha=0.1$ & 0.058 & -0.042 & 1.968 & 0.056 & 5.754 & 0.386 & IV \\
& $\beta=0.2$ & 0.391 & 0.191 & & 0.319 & & 0.79 & IV
\end{tabular}




\begin{tabular}{|c|c|c|c|c|c|c|c|c|}
\hline & $\theta=0.5$ & 0.575 & 0.075 & & 0.263 & & 0.372 & IV \\
\hline & $\lambda=4$ & 4.846 & 0.846 & & 4.86 & & 0.407 & IV \\
\hline & $c=3$ & 1.235 & -1.765 & & 3.052 & & -0.562 & I \\
\hline \multirow[t]{5}{*}{20} & $\alpha=0.1$ & 0.078 & -0.022 & 1.544 & 0.055 & 4.524 & 0.302 & IV \\
\hline & $\beta=0.2$ & 0.369 & 0.169 & & 0.085 & & 0.24 & IV \\
\hline & $\theta=0.5$ & 0.565 & 0.065 & & 0.083 & & -0.172 & I \\
\hline & $\lambda=4$ & 4.743 & 0.743 & & 3.423 & & 0.617 & IV \\
\hline & $c=3$ & 1.658 & -1.342 & & 2.955 & & 0.399 & IV \\
\hline \multirow[t]{5}{*}{30} & $\alpha=0.1$ & 0.085 & -0.015 & 1.125 & 0.056 & 4.02 & 0.244 & IV \\
\hline & $\beta=0.2$ & 0.316 & 0.116 & & 0.038 & & 0.875 & IV \\
\hline & $\theta=0.5$ & 0.543 & 0.043 & & 0.069 & & 0.905 & IV \\
\hline & $\lambda=4$ & 4.576 & 0.576 & & 2.712 & & 0.715 & IV \\
\hline & $c=3$ & 2.041 & -0.959 & & 2.966 & & 0.518 & IV \\
\hline \multirow[t]{5}{*}{50} & $\alpha=0.1$ & 0.093 & -0.007 & 0.578 & 0.057 & 3.641 & 0.319 & IV \\
\hline & $\beta=0.2$ & 0.283 & 0.083 & & 0.032 & & 0.105 & IV \\
\hline & $\theta=0.5$ & 0.528 & 0.028 & & 0.062 & & -2.276 & I \\
\hline & $\lambda=4$ & 4.334 & 0.334 & & 2.108 & & 0.618 & IV \\
\hline & $c=3$ & 2.536 & -0.464 & & 2.967 & & -0.579 & I \\
\hline \multirow[t]{5}{*}{100} & $\alpha=0.1$ & 0.096 & -0.004 & 0.180 & 0.061 & 3.502 & -0.399 & I \\
\hline & $\beta=0.2$ & 0.247 & 0.047 & & 0.031 & & 0.149 & IV \\
\hline & $\theta=0.5$ & 0.513 & 0.013 & & 0.059 & & 4.216 & VI \\
\hline & $\lambda=4$ & 4.119 & 0.119 & & 1.872 & & 0.346 & IV \\
\hline & $c=3$ & 2.874 & -0.126 & & 2.959 & & 0.709 & IV \\
\hline \multirow[t]{5}{*}{300} & $\alpha=0.1$ & 0.097 & -0.003 & 0.075 & 0.037 & 1.393 & 0.105 & IV \\
\hline & $\beta=0.2$ & 0.205 & 0.005 & & 0.048 & & 0.643 & IV \\
\hline & $\theta=0.5$ & 0.504 & 0.004 & & 0.024 & & -0.57 & I \\
\hline & $\lambda=4$ & 4.071 & 0.071 & & 0.618 & & 0.671 & IV \\
\hline & $c=3$ & 2.976 & -0.024 & & 1.247 & & 0.347 & IV \\
\hline
\end{tabular}

